\title{
Defining characteristics and related factors of the nursing diagnosis for ineffective breathing pattern
}

\author{
Características definidoras e fatores relacionados do diagnóstico de enfermagem padrão respiratório ineficaz \\ Características definitorias y factores relacionados del diagnóstico de enfermería estándar respiratorio ineficaz
}

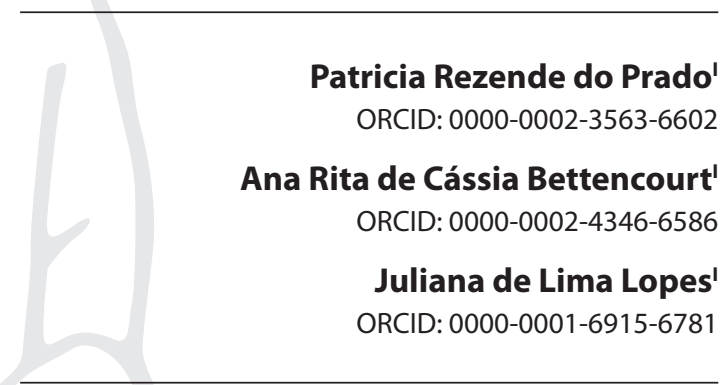

'Universidade Federal de São Paulo, Paulista Nursing School. São Paulo, São Paulo, Brazil.

How to cite this article: Prado PR, Bettencourt ARC, Lopes JL. Defining characteristics and related factors of the nursing diagnosis for ineffective breathing pattern.

Rev Bras Enferm [Internet]. 2019;72(1):221-30. DOI: http://dx.doi.org/10.1590/0034-7167-2018-0061

Corresponding Author:

Patricia Rezende do Prado E-mail: patyrezendeprado@gmail.com

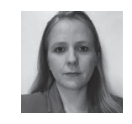

Submission: 02-06-2018

Approval: 04-21-2018

\section{ABSTRACT}

Objective: To identify in the literature the defining characteristics and related factors of the nursing diagnosis "ineffective breathing pattern". Method: Integrative review with the steps: problem identification, literature search, evaluation and analysis of data and presentation of results. Results: Twenty articles and two dissertations were included. In children, the most prevalent related factor was bronchial secretion, followed by hyperventilation. The main defining characteristics were dyspnea, tachypnea, cough, use of accessory muscles to breathe, orthopnea and adventitious breath sounds. Bronchial secretion, cough and adventitious breath sounds are not included in the NANDA-International (NANDA-I). For adults and older adults, the related factors were fatigue, pain and obesity and the defining characteristics were dyspnea, orthopnea and tachypnea. Conclusion: This diagnosis manifests differently according to the patients' age group. It was observed that some defining characteristics and related factors are not included in the NANDA-I. Their inclusion can improve this nursing diagnosis.

Descriptors: Nursing Diagnosis; Respiratory System; Signs and Symptoms; Risk Factors; Nursing.

\section{RESUMO}

Objetivo: Identificar na literatura as características definidoras e os fatores relacionados do diagnóstico de enfermagem Padrão respiratório ineficaz. Método: Revisão integrativa com as etapas formulação do problema, revisão da literatura, avaliação e análise dos dados e apresentação de resultados. Resultados: Foram incluídos 20 artigos e 02 dissertações. Em crianças, o fator relacionado mais prevalente nos estudos foi a secreção nos brônquios, seguido da hiperventilação. As principais características definidoras foram dispneia, taquipneia, tosse, uso da musculatura acessória para respirar, ortopneia e ruídos adventícios, sendo que a secreção nos brônquios, a tosse e os ruídos adventícios não constam na NANDA-Internacional (NANDA-I). Para adultos e idosos, os fatores relacionados foram fadiga, dor e obesidade. As características definidoras foram dispneia, ortopneia e taquipneia. Conclusão: Esse diagnóstico manifesta-se diferententemente de acordo com a faixa etária dos pacientes. Observouse que algumas características definidoras e fator relacionado não constam na NANDA-I, cuja inclusão pode aprimorar esse diagnóstico de enfermagem.

Descritores: Diagnóstico de Enfermagem; Sistema Respiratório; Sinais e Sintomas; Fatores de Risco; Enfermagem.

\section{RESUMEN}

Objetivo: Identificar en la literatura las características definitorias y los factores relacionados del diagnóstico de enfermería estándar respiratorio ineficaz. Método: Se trata de una revisión integradora conteniendo las etapas de formulación del problema, revisión da literatura, evaluación y análisis de los datos y presentación de los resultados. Resultados: Se incluyeron 20 artículos y 02 disertaciones. En los niños, el factor relacionado que prevaleció fue la secreción en los bronquios seguido de hiperventilación. Las características definitorias principales fueron la disnea, la taquipnea, la tos, el uso de la musculatura accesoria para respirar, la ortopnea y los ruidos adventicios, siendo que la secreción en los bronquios, la tos y los ruidos adventicios no constan en la NANDAInternacional (NANDA-I). Para los adultos y personas mayores, los factores relacionados fueron la fatiga, el dolor y la obesidad, y las características definitorias, la disnea, la ortopnea y la taquipnea. Conclusión: Este diagnóstico difiere según la edad de los pacientes; cabe destacar que ciertas características definitorias y factores relacionados no constan en la NANDA-I, cuya inclusión podría mejorar este diagnóstico de enfermería. Descriptores: Diagnóstico de Enfermería; Sistema Respiratorio; Signos y Síntomas; Factores de Riesgo; Enfermería. 


\section{INTRODUCTION}

The main function of the respiratory system is to perform gas exchange. It extracts oxygen from the external environment, transports it to the lungs, exchanges carbon dioxide in the alveoli and expels it back into the environment ${ }^{(1)}$.

When there is impairment of the lungs, respiratory or accessory muscles, the body does not receive enough oxygen for the cells, leading to an abnormal respiratory pattern. There are several possible causes for this phenomenon, such as heart failure, airway obstruction, diaphragm paralysis, infection, neuromuscular deficiency, trauma or surgery ${ }^{(1)}$.

Nursing professionals contribute significantly to the identification of patients with respiratory problems, through anamnesis and physical examination. This evaluation favors the identification of care needs, with the definition of nursing diagnosis and, later, of nursing interventions ${ }^{(1-2)}$.

The use of Nursing Diagnoses (ND) brings the nurses closer to the patients and requires clinical reasoning and analysis of the signs and symptoms (defining characteristics) and the causes (related factors) identified in the physical examination and anamnesis. Diagnosis involves a cognitive process, in which the practitioner makes inferences about the observed data, which, if interpreted correctly, allows directing actions and predicting the desired results, thus improving the quality of nursing care ${ }^{(2)}$.

Given the above, great efforts are being made to standardize and improve the classification of nursing diagnoses. Since 1973, NANDA-International (NANDA-I) has been working to standardize nursing diagnostic terminology ${ }^{(3)}$ and one of its diagnoses is Ineffective Breathing Pattern, defined as inspiration and/or expiration that does not provide adequate ventilation ${ }^{(3)}$.

This diagnosis has a high prevalence among children and adults, as it is identified in $86.7 \%$ of children with congenital heart disease ${ }^{(4)}$ and $85.7 \%$ of adults in emergency services ${ }^{(5)}$. Due to this high prevalence, it is fundamental to conduct studies that identify the defining characteristics and related factors of this ND.

\section{OBJECTIVE}

To identify in the literature the defining characteristics and related factors of the nursing diagnosis "ineffective breathing pattern".

\section{METHOD}

The integrative literature review was conducted according to the model described by Whittemore \& Knafl's ${ }^{(6)}$, with the following stages: problem identification, literature search, evaluation, analysis of data and presentation of results.

The searches were conducted at the following databases: Cochrane, JBC Conect, Scopus, Science Direct, Lilacs and Medline. The inclusion criteria were: articles or dissertations/theses published in Portuguese, English and Spanish, between 1990 and 2016 and containing the characteristics and/or related factors of the nursing diagnosis for ineffective breathing pattern in patients on spontaneous ventilation. The exclusion criteria were review and meta-analysis articles. The guiding question of this review was: What are the defining characteristics and/or related factors of patients on spontaneous ventilation with the nursing diagnosis Ineffective Breathing Pattern?

The descriptors and terms used were: signals and symptoms (sinais e sintomas and signos y síntomas), nursing diagnosis (diagnósticos de enfermagem and diagnóstico de enfermería), ineffective breathing pattern (padrão respiratório ineficaz and patrón respiratorio ineficaz), defining characteristics (características definidoras e características definidoras), risk factors (fatores de risco and factores de riesgo), related factors (fatores relacionados and factores relacionados) and pulmonary ventilation (ventilação pulmonar and ventilación pulmonar). The search was conducted using the following Boolean operators: (nursing diagnosis AND ineffective breathing pattern) OR (pulmonary ventilation) AND (signals and symptoms OR defining characteristics OR risk factors OR related factors).

The search was conducted by online access and the abstracts were independently evaluated by two researchers and those that reached consensus were selected for full reading. The articles/ theses selected were read in full by the two researchers and those that met the inclusion criteria were included. The final sample was composed of 20 articles and 02 dissertations (Figure 1).

Data was extracted according to an instrument elaborated by the researchers with the following items: title of the article, author, quality of the study, journal, objective, study type, location of study, sample and results.

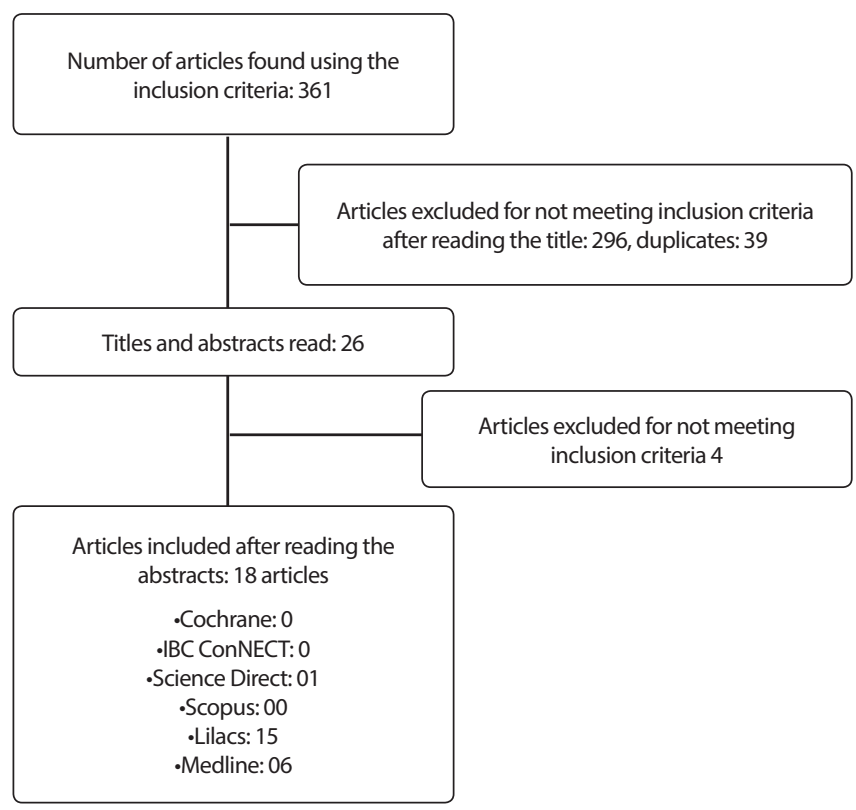

Figure 1 - Overview of the integrative review process

The methodological quality of the studies was evaluated by the Newcastle-Ottawa Scale, which assesses non-randomized studies, including case-control, cohort and cross-sectional studies. This scale is a continuous collaboration between the Universities of Newcastle, Australia, Ottawa and Canada. A 'star system' has been developed in which a study is judged on three broad perspectives: the selection of the study groups; the comparability of the groups; and the presentation and discussion of the data obtained, which should allow the reader to evaluate the applicability of the integrative review in order to achieve the objective 
of this method ${ }^{(7)}$. For cross-sectional studies, the subscales are: representativeness of sample (maximum 5 stars), comparability ( 2 stars) and outcomes (3 stars). For cohort studies, the subscales are: selection of the cohort (6 stars), comparability of cohort (2 stars) and outcome (5 stars). For case-control studies, the subscales are: selection of cases and controls (4 stars), comparability of cases and controls ( 2 stars) and ascertainment of exposure ( 4 stars). Stars are converted into points and studies with more than six points were considered of good methodological quality ${ }^{(7)}$.

\section{RESULTS}

Twenty articles ${ }^{(8-27)}$ and 2 dissertations ${ }^{(28-29)}$ were identified, one from Science Direct, six from Medline and 15 from Lilacs (Figure 1).

Regarding the journal, $13.6 \%$ of the studies were published in the University of São Paulo Nursing School Journal, 13.6\% in the Brazilian Journal of Nursing, $13.6 \%$ in the Journal of Northeastern Nursing Network, 9,1\% in the Journal of Clinical Nursing and only one study (4,55\%) was identified in each of the following journals: Journal of Pediatric Nursing, Applied Nursing Research, CHIA, Journal of Nursing Scholarship, International Journal of Nursing
Knowledge, Latin American Journal of Nursing, Acta Paulista Nursing, Revista Eletrônica de Enfermagem, Cogitare Nursing. It was also observed that $9.1 \%$ of the studies were dissertations.

Regarding the study design, 18 (81.8\%) were cross-sectional, three $(13,6 \%)$ were cohort studies and one was a clinical validation study (4.6\%). Regarding the location of the studies, only one was conducted in the United States (4.5\%) and the other $95.5 \%$ were developed in Brazil (Chart 1 and 2).

After reading, articles/dissertations were separated into two categories: 1) Ineffective breathing pattern in children and 2) Ineffective breathing pattern in adults and older adults. Eleven studies were conducted with children and eleven with adults and older adults, and $100.0 \%$ of studies with children were conducted in Brazil (Chart 1 and 2).

In children, the most prevalent related factor found in the studies was bronchial secretion, followed by hyperventilation. The main defining characteristics were: dyspnea, tachypnea, cough, use of accessory muscles to breathe, orthopnea and adventitious breath sounds (Chart 1). In adults and older adults, the main related factors were: fatigue, pain and obesity. The main defining characteristics were dyspnea, tachypnea and orthopnea (Chart 2).

Chart 1 - Articles from the literature review on IBP in children, 1980-2016

\begin{tabular}{|c|c|c|c|c|}
\hline Authors and year & Quality of the study & Study type & Sample & Results \\
\hline $\begin{array}{c}\text { Monteiro, } \\
\text { Silva and } \\
\text { Lopes }(2006)^{(8)}\end{array}$ & $\begin{array}{l}\text { Good ( } 6 \text { out of } \\
10 \text { points in the } \\
\text { Newcastle-Ottawa } \\
\text { scale for cross- } \\
\text { sectional studies). }\end{array}$ & $\begin{array}{l}\text { Cross-sectional } \\
\text { study. }\end{array}$ & $\begin{array}{l}78 \text { children under } \\
5 \text { years old. }\end{array}$ & $\begin{array}{l}\text { DC: } \\
\text { - Adventitious breath sounds }(96.2 \%, n=75) \text {. } \\
\text { - Dyspnea }(74.4 \%, n=58) \text {. } \\
\text { - Shortness of breath }(69.2 \%, n=54) \text {. } \\
\text { - Cough }(60.3 \%, n=47) \text {. } \\
\text { - Changes in respiratory rate and pattern }(59.0 \%, n=46) \text {. } \\
\text { - Use of accessory muscles to breathe }(25.6 \%, n=20) \text {. } \\
\text { RF: } \\
\text { - Bronchial secretion }(98.7 \%, n=77) \text {. } \\
\text { - Hyperventilation }(74.4 \%, n=58) .\end{array}$ \\
\hline $\begin{array}{c}\text { Silveira, } \\
\text { Lima and Lopes } \\
(2008)^{(9)}\end{array}$ & $\begin{array}{l}\text { Good (6 out of } \\
10 \text { points in the } \\
\text { Newcastle-Ottawa } \\
\text { scale for cross- } \\
\text { sectional studies). }\end{array}$ & $\begin{array}{l}\text { Cross-sectional } \\
\text { study. }\end{array}$ & $\begin{array}{l}42 \text { children up } \\
\text { to } 5 \text { years old } \\
\text { with diagnosis of } \\
\text { asthma. }\end{array}$ & $\begin{array}{l}\text { DC: } \\
\text { - Tachypnea }(61.9 \%, n=26) \text {. } \\
\text { - Dyspnea }(50.0 \%, n=21) \text {. } \\
\text { - Orthopnea }(40.5 \%, n=17) \text {. } \\
\text { - Use of accessory muscles to breathe }(35.7 \%, n=15) \text {. } \\
\text { - Nasal flaring }(4.8 \%, n=2) \text {. } \\
\text { - Increase in anterior-posterior chest diameter }(4.8 \%, n=2) \text {. } \\
\text { - Altered chest excursion }(2.4 \%, n=1) \text {. } \\
\text { - Pursed-lip breathing }(2.4 \%, n=1) \text {. }\end{array}$ \\
\hline $\begin{array}{c}\text { Cavalcante, } \\
\text { Mendes, Lopes } \\
\text { and Lima }(2010)^{(10)}\end{array}$ & $\begin{array}{l}\text { Good (6 out of } \\
10 \text { points in the } \\
\text { Newcastle-Ottawa } \\
\text { scale for cross- } \\
\text { sectional studies). }\end{array}$ & $\begin{array}{l}\text { Cross-sectional } \\
\text { study. }\end{array}$ & $\begin{array}{l}147 \text { children with } \\
\text { asthma. }\end{array}$ & $\begin{array}{l}\text { DC: } \\
\text { - Dyspnea (56.4\%, } \mathrm{n}=83) \text {. } \\
\text { - Abnormal breathing pattern }(50.3 \%, \mathrm{n}=74) \text {. } \\
\text { - Orthopnea }(34.0 \%, \mathrm{n}=50) \text {. } \\
\text { - Use of accessory muscles to breathe }(32.7 \%, \mathrm{n}=48) \text {. } \\
\text { - Increase in anterior-posterior chest diameter }(8.16 \%, \mathrm{n}=12) \text {. } \\
\text { - Nasal flaring }(3.4 \%, \mathrm{n}=5) \text {. } \\
\text { - Pursed-lip breathing }(3.4 \%, \mathrm{n}=5) \text {. } \\
\text { - Use of three-point position }(2.04 \%, \mathrm{n}=3) \text {. } \\
\text { - Altered chest excursion }(2.04 \%, \mathrm{n}=3) \text {. }\end{array}$ \\
\hline $\begin{array}{c}\text { Chagas, Lima, } \\
\text { Oliveira and Luz } \\
(2011)^{(11)}\end{array}$ & $\begin{array}{l}\text { Good ( } 6 \text { out of } \\
10 \text { points in the } \\
\text { Newcastle-Ottawa } \\
\text { scale for cross- } \\
\text { sectional studies). }\end{array}$ & $\begin{array}{l}\text { Cross-sectional } \\
\text { study. }\end{array}$ & $\begin{array}{c}30 \text { children up to } 5 \\
\text { years old. }\end{array}$ & $\begin{array}{l}\text { DC: } \\
\quad \text { - Dyspnea }(96.7 \%, \mathrm{n}=29) \text {. } \\
\text { - Altered breathing depth }(73.3 \%, \mathrm{n}=22) \text {. } \\
\text { - Use of accessory muscles to breathe }(56.7 \%, \mathrm{n}=17) \text {. } \\
\text { - Tachypnea }(33.3 \%, \mathrm{n}=10) \\
\text { - Nasal flaring }(6.7 \%, \mathrm{n}=2) \\
\text { RF: } \\
\text { - Respiratory muscle fatigue }(73.3 \%, \mathrm{n}=22) \\
\text { - Obesity }(3.3 \%, \mathrm{n}=1) \text {. }\end{array}$ \\
\hline
\end{tabular}




\begin{tabular}{|c|c|c|c|c|}
\hline Authors and year & Quality of the study & Study type & Sample & Results \\
\hline $\begin{array}{c}\text { Andrade, Chaves, } \\
\text { Silva, } \\
\text { Beltrão and } \\
\text { Lopes }(2012)^{(12)}\end{array}$ & $\begin{array}{l}\text { Good ( } 7 \text { out of } \\
10 \text { points in the } \\
\text { Newcastle-Ottawa } \\
\text { scale for cross- } \\
\text { sectional studies). }\end{array}$ & $\begin{array}{l}\text { Cross-sectional } \\
\text { study. }\end{array}$ & $\begin{array}{l}151 \text { children up to } \\
5 \text { years old. }\end{array}$ & $\begin{array}{l}\text { DC: } \\
\text { - Adventitious breath sounds }(77.5 \%, \mathrm{n}=117) \text {. } \\
\text { - Cough }(70.2 \%, \mathrm{n}=106) \text {. } \\
\text { - Dyspnea }(55.6 \%, \mathrm{n}=84) \text {. } \\
\text { - Tachypnea }(54.3 \%, \mathrm{n}=82) \text {. } \\
\text { - Abnormal breathing pattern }(54.3 \%, \mathrm{n}=82) \text {. } \\
\text { - Use of accessory muscles to breathe }(43.7 \%, \mathrm{n}=66) \text {. } \\
\text { - Orthopnea }(32.5 \%, \mathrm{n}=49) \text {. } \\
\text { - Nasal flaring }(3.3 \%, \mathrm{n}=5) \text {. } \\
\text { - Pursed-lip breathing }(0.7 \%, \mathrm{n}=1) \text {. } \\
\text { RF: } \\
\text { - Bronchial secretion }(89.4 \%, \mathrm{n}=135) \text {. } \\
\text { - Anxiety }(22.5 \%, \mathrm{n}=34) \\
\text { - Pain }(16.6 \%, \mathrm{n}=25) \text {. } \\
\text { - Chest wall deformity }(2.6 \%, \mathrm{n}=4) \text {. }\end{array}$ \\
\hline $\begin{array}{l}\text { Pascoal, Lopes, } \\
\text { da Silva, Beltrão, } \\
\text { Chaves, Santiago } \\
\text { and Herdman } \\
(2014)^{(13)}\end{array}$ & $\begin{array}{l}\text { Good ( } 6 \text { out of } \\
10 \text { points in the } \\
\text { Newcastle-Ottawa } \\
\text { scale for cross- } \\
\text { sectional studies). }\end{array}$ & $\begin{array}{l}\text { Prospective } \\
\text { cohort study. }\end{array}$ & $\begin{array}{l}136 \text { children } \\
\text { from } 0 \text { to } 5 \text { years } \\
\text { old with acute } \\
\text { respiratory } \\
\text { infection. }\end{array}$ & $\begin{array}{l}\text { DC: } \\
\text { - Altered breathing depth }(75 \%, n=99) \text {. } \\
\text { - Dyspnea }(75 \%, n=99) \text {. } \\
\text { - Orthopnea }(61 \%, n=80.0) \text {. } \\
\text { - Tachypnea }(61 \%, n=80.0) \text {. } \\
\text { - Use of accessory muscles to breathe }(61 \%, n=80.0) \text {. }\end{array}$ \\
\hline $\begin{array}{l}\text { Beltrão, } \\
\text { Herdman, } \\
\text { Pascoal, Chaves, } \\
\text { Silva, and Lopes } \\
\quad(2015)^{(14)}\end{array}$ & $\begin{array}{l}\text { Good ( } 6 \text { out of } \\
10 \text { points in the } \\
\text { Newcastle-Ottawa } \\
\text { scale for cross- } \\
\text { sectional studies). }\end{array}$ & $\begin{array}{l}\text { Cross-sectional } \\
\text { study. }\end{array}$ & $\begin{array}{l}61 \text { children and } \\
\text { adolescents }(5 \\
\text { to } 17 \text { years) with } \\
\text { congenital heart } \\
\text { disease. }\end{array}$ & $\begin{array}{l}\text { DC: } \\
\text { - Dyspnea }(45.9 \%, n=28) \text {. } \\
\text {-Altered breathing depth }(36.1 \%, \mathrm{n}=22) \text {. } \\
\text { - Orthopnea }(27.9 \%, \mathrm{n}=17) \text {. } \\
\text { - Tachypnea }(19.7 \%, \mathrm{n}=12) \text {. } \\
\text { - Use of accessory muscles to breathe }(18.0 \%, \mathrm{n}=11) \text {. } \\
\text { - Increase in anterior-posterior chest diameter }(18.0 \%, \mathrm{n}=11) \text {. } \\
\text { - Use of three-point position }(6.6 \%, \mathrm{n}=4) \text {. } \\
\text { - Bradypnea }(4.9 \%, \mathrm{n}=3) \text {. }\end{array}$ \\
\hline $\begin{array}{l}\text { Pascoal, Lopes, } \\
\text { Silva, Beltrão, } \\
\text { Chaves, Nunes } \\
\text { and Castro } \\
(2016)^{(15)}\end{array}$ & $\begin{array}{l}\text { Good ( } 7 \text { out of } \\
10 \text { points in the } \\
\text { Newcastle-Ottawa } \\
\text { scale for cohort } \\
\text { studies). }\end{array}$ & $\begin{array}{l}\text { Prospective } \\
\text { cohort study }\end{array}$ & 136 children. & $\begin{array}{l}\text { DC: } \\
\text { - Altered breathing depth }(66.3 \%, n=65) \text {. } \\
\text { - Nasal flaring }(4.0 \%, n=4) \text {. } \\
\text { - Bradypnea }(1.0 \%, n=0,76) \text {. } \\
\text { - Increase in anterior-posterior chest diameter }(26,8 \%, n=30) \text {. } \\
\text { - Dyspnea (43.1\%; } \mathrm{n}=51) \text {. } \\
\text { - Altered chest excursion }(1,5 \%, n=1.7) \text {. } \\
\text { - Orthopnea }(31.5 ; n=37.8) \text {. } \\
\text { - Pursed-lip breathing }(1.45 \%, n=2) \text {. } \\
\text { - Tachypnea }(55.3 \%, n=63) \text {. } \\
\text { - Use of accessory muscles to breathe: }(36.9 \%, n=43.3) \text {. }\end{array}$ \\
\hline $\begin{array}{c}\text { Pascoal, Lopes, } \\
\text { Silva, Chaves, } \\
\text { Beltrão, Nunes } \\
\text { and Moura (2016) } \\
\underset{(16)}{ }\end{array}$ & $\begin{array}{l}\text { Good ( } 7 \text { out of } \\
10 \text { points in the } \\
\text { Newcastle-Ottawa } \\
\text { scale for cohort } \\
\text { studies). }\end{array}$ & Cohort study. & $\begin{array}{l}136 \text { children with } \\
\text { acute respiratory } \\
\text { infection. }\end{array}$ & $\begin{array}{l}\text { DC: } \\
\text { - Cough (88.7\%). } \\
\text { - Abnormal breathing (68.7\%). } \\
\text { - Adventitious breath sounds (65.1\%). } \\
\text { - Tachypnea (55.8\%). } \\
\text { - Dyspnea (45.7\%). } \\
\text { - Use of accessory muscles to breathe (38.4\%). } \\
\text { - Orthopnea (34.1\%). } \\
\text { - Diminished vesicular breath sounds (34.0\%). } \\
\text {-Increase in anterior-posterior chest diameter(27.7\%). }\end{array}$ \\
\hline Chaves $(2011)^{(28)}$ & $\begin{array}{l}\text { Good ( } 8 \text { out of } \\
10 \text { points in the } \\
\text { Newcastle-Ottawa } \\
\text { scale for cross- } \\
\text { sectional studies). }\end{array}$ & $\begin{array}{l}\text { Cross-sectional } \\
\text { study. }\end{array}$ & $\begin{array}{l}249 \text { children with } \\
\text { diagnosis of ARF. }\end{array}$ & $\begin{array}{l}\text { DC: } \\
\text { - Altered breathing depth }(73.9 \%, \mathrm{n}=184) \text {. } \\
\text { - Dyspnea }(68.3 \%, \mathrm{n}=170) \text {. } \\
\text { - Tachypnea }(57.0 \%, \mathrm{n}=142) \text {. } \\
\text { - Orthopnea }(54.2 \%, \mathrm{n}=135) \text {. } \\
\text { - Use of accessory muscles to breathe }(51.8 \%, \mathrm{n}=129) \text {. }\end{array}$ \\
\hline Carvalho $(2013)^{(29)}$ & $\begin{array}{l}\text { Good (7 out of } \\
10 \text { points in the } \\
\text { Newcastle-Ottawa } \\
\text { scale for cross- } \\
\text { sectional studies). }\end{array}$ & $\begin{array}{l}\text { Cross-sectional } \\
\text { study. }\end{array}$ & $\begin{array}{l}205 \text { children. From } \\
01 \text { to } 05 \text { years old. }\end{array}$ & $\begin{array}{l}\text { DC: } \\
\text { - Altered breathing depgh }(91.2 \%, n=187) \text {. } \\
\text { - Use of three-point position }(1 \%, n=2) \text {. } \\
\text { - Nasal flaring ( } 3.4 \%, n=7) \text {. } \\
\text { - Pursed-lip breathing }(0.5 \%, n=1) \text {. } \\
\text { - Dyspnea ( } 99 \%, n=203) \text {. } \\
\text { - Altered chest excursion }(1 \%, n=2) \\
\text { - Orthopnea }(91.2 \%, n=187) \text {. } \\
\text { - Tachypnea }(85.4 \%, n=175) \text {. } \\
\text { - Use of accessory muscles to breathe }(84.9 \%, n=174) \text {. }\end{array}$ \\
\hline
\end{tabular}


Chart 2 - Articles from the literature review on IBP in adults, 1980-2016

\begin{tabular}{|c|c|c|c|c|}
\hline Authors and year & Quality of the study & Type of study & Sample & Results \\
\hline $\begin{array}{l}\text { Carlson-Catalano, } \\
\text { Lunney, Paradiso, } \\
\text { Bruno, Luke, Martin, } \\
\text { Massoni and } \\
\text { Pachter (1998) })^{(17)}\end{array}$ & - & $\begin{array}{l}\text { Clinical } \\
\text { validation. }\end{array}$ & $\begin{array}{c}76 \text { adult patients, } \\
45 \text { medical and } 31 \\
\text { surgical. }\end{array}$ & $\begin{array}{l}\text { DC: } \\
\text { - Dyspnea. } \\
\text { RF: } \\
\text { - Anxiety }(15 \%, n=8) \text {. } \\
\text { - Pain }(17 \%, n=9) \\
\text { - Fatigue }(38 \%, n=20) \text {. } \\
\text { - Hyperventilation }(8 \%, n=4) \text {. } \\
\text { - Obesity }(4 \%, n=2) \text {. } \\
\text { - Musculoskeletal impairment }(6 \%, n=3) \text {. } \\
\text { - Bronchial secretion }(21 \%, n=11) \text {. }\end{array}$ \\
\hline $\begin{array}{l}\text { Arruda and Garcia } \\
\qquad(2000)^{(18)}\end{array}$ & $\begin{array}{l}\text { Good ( } 6 \text { out of } \\
10 \text { points in the } \\
\text { Newcastle-Ottawa } \\
\text { scale for cross- } \\
\text { sectional studies). }\end{array}$ & $\begin{array}{l}\text { Cross-sectional } \\
\text { study. }\end{array}$ & $\begin{array}{c}22 \\
\text { patients. }\end{array}$ & $\begin{array}{l}\text { DC: } \\
\quad \text { - Tachypnea }(31.8 \%, n=7) \\
\text { - Dyspnea }(9.1 \%, n=2) \\
\text { RF: } \\
\text { - Anxiety }(35.3 \%, n=6) \\
\text { - Hypoventilation syndrome }(17.6 \%, n=3) \text {. }\end{array}$ \\
\hline $\begin{array}{l}\text { Galdeano, Rossi } \\
\text { and Pezzuto } \\
(2004)^{(19)}\end{array}$ & $\begin{array}{l}\text { Good ( } 6 \text { out of } \\
10 \text { points in the } \\
\text { Newcastle-Ottawa } \\
\text { scale for cross- } \\
\text { sectional studies). }\end{array}$ & $\begin{array}{l}\text { Cross-sectional } \\
\text { study. }\end{array}$ & 17 adults. & $\begin{array}{l}\text { DC: } \\
\text { - Dyspnea }(100.0 \%, n=12) \text {. } \\
\text { - Orthopnea }(66.6 \%, n=8) \text {. } \\
\text { - Shortness of breath }(16.6 \%, n=2) \text {. } \\
\text { RF: } \\
\text { - Decreased energy/fatigue }(100.0 \%, n=12) \text {. } \\
\text { - Body position }(66.6 \%, n=8) \text {. } \\
\text { - Obesity }(33.3 \%, n=4) \text {. }\end{array}$ \\
\hline $\begin{array}{l}\text { Oliveira and } \\
\text { Freitas }(2009)^{(20)}\end{array}$ & $\begin{array}{l}\text { Good ( } 6 \text { out of } \\
10 \text { points in the } \\
\text { Newcastle-Ottawa } \\
\text { scale for cross- } \\
\text { sectional studies). }\end{array}$ & $\begin{array}{l}\text { Cross-sectional } \\
\text { study. }\end{array}$ & 28 charts. & $\begin{array}{l}\text { DC: } \\
\quad \text { - Irregular minute ventilation }(71.4 \%, n=20) \text {. } \\
\text { - Dyspnea }(71.4 \%, n=20) \\
\text { - Increased chest excursion }(71.4 \%, n=20)\end{array}$ \\
\hline $\begin{array}{l}\text { Silva, Bettencourt, } \\
\text { Diccini, } \\
\text { Belasco and } \\
\text { Barbosa }(2009)^{(21)}\end{array}$ & $\begin{array}{l}\text { Good ( } 6 \text { out of } \\
10 \text { points in the } \\
\text { Newcastle-Ottawa } \\
\text { scale for cross- } \\
\text { sectional studies). }\end{array}$ & $\begin{array}{l}\text { Cross-sectional } \\
\text { study. }\end{array}$ & 60 patients & $\begin{array}{ll}\text { DC: } & \\
& \text { - Decrease in expiratory and inspiratory pressure } \\
& (70.0 \%, \mathrm{n}=42) . \\
& \text { - Dyspnea }(51.6 \%, \mathrm{n}=31) . \\
\text { RF: } & \\
& \text { - Hypoventilation syndrome }(51.6 \%, \mathrm{n}=31) . \\
& \text { - Neurological/perception impairment }(45.0 \%, \mathrm{n}=27) \text {. } \\
& \text { - Neuromuscular dysfunction }(40.0 \%, \mathrm{n}=24) .\end{array}$ \\
\hline $\begin{array}{l}\text { Lucena, Santos, } \\
\text { Pereira, Almeida, } \\
\text { Dias and Friedrich } \\
(2011)^{(22)}\end{array}$ & $\begin{array}{l}\text { Good ( } 6 \text { out of } \\
10 \text { points in the } \\
\text { Newcastle-Ottawa } \\
\text { scale for cross- } \\
\text { sectional studies). }\end{array}$ & $\begin{array}{l}\text { Cross-sectional } \\
\text { study. }\end{array}$ & $\begin{array}{l}194 \text { hospitalizations } \\
\text { of adult patients in } \\
\text { clinical and surgical } \\
\text { units at risk of } \\
\text { developing PU. }\end{array}$ & $\begin{array}{l}\text { RF: } \\
\text { - Neuromuscular and/or musculoskeletal impairment } \\
(34.0 \%, n=18) .\end{array}$ \\
\hline $\begin{array}{l}\text { Moreira, Caetano, } \\
\text { Barros and Galvão } \\
\quad(2013)^{(23)}\end{array}$ & $\begin{array}{l}\text { Good ( } 7 \text { out of } \\
10 \text { points in the } \\
\text { Newcastle-Ottawa } \\
\text { scale for cross- } \\
\text { sectional studies). }\end{array}$ & $\begin{array}{l}\text { Cross-sectional } \\
\text { study. }\end{array}$ & $\begin{array}{l}20 \text { patients after } \\
\text { bariatric surgery. }\end{array}$ & $\begin{aligned} \text { RF: } & \\
& \text { - Obesity }(100.0 \%, n=20) \\
& \text { - Fatigue }(55.0 \%, n=11) \\
& \text { - Respiratory muscle fatigue }(45.0 \%, n=9) \\
& \text { - Anxiety }(10.0 \%, n=2)\end{aligned}$ \\
\hline $\begin{array}{l}\text { Souza, Mata, } \\
\text { Carvalho and } \\
\text { Chianca }(2013)^{(24)}\end{array}$ & $\begin{array}{l}\text { Good ( } 7 \text { out of } \\
10 \text { points in the } \\
\text { Newcastle-Ottawa } \\
\text { scale for cross- } \\
\text { sectional studies). }\end{array}$ & $\begin{array}{l}\text { Cross-sectional } \\
\text { study. }\end{array}$ & 40 charts. & $\begin{aligned} \text { DC: } & \\
& \text { - Tachypnea }(100.0 \%, n=40) . \\
& \text { - Dyspnea }(66.7 \%, n=27) \text {. } \\
& \text { - Use of accessory muscles to breathe }(22.2 \%, n=9) . \\
\text { RF: } & \\
& \text { - Hyperventilation }(100.0 \%, n=40) \\
& \text { - Pain }(77.8 \%, n=31) .\end{aligned}$ \\
\hline $\begin{array}{l}\text { Barros, Moreira, } \\
\text { Frota and Caetano } \\
(2015)^{(25)}\end{array}$ & $\begin{array}{l}\text { Good ( } 7 \text { out of } \\
10 \text { points in the } \\
\text { Newcastle-Ottawa } \\
\text { scale for cross- } \\
\text { sectional studies). }\end{array}$ & $\begin{array}{l}\text { Cross-sectional } \\
\text { study. }\end{array}$ & 58 adult patients. & $\begin{aligned} \text { DC: } & \\
& \text { - Dyspnea }(68.2 \%, \mathrm{n}=39) . \\
& \text { - Orthopnea }(59.0 \%, \mathrm{n}=34) \\
\text { RF: } & \\
& \text { - Obesity }(86.4 \%, \mathrm{n}=50) \\
& \text { - Fatigue }(77.3 \%, \mathrm{n}=45) \\
& \text { - Pain }(63.6 \%, \mathrm{n}=37) \\
& \text { - Respiratory muscle fatigue }(4.5 \%, \mathrm{n}=3) . \\
& \text { - Anxiety }(4.5 \%, \mathrm{n}=3) .\end{aligned}$ \\
\hline
\end{tabular}




\begin{tabular}{|c|c|c|c|c|}
\hline Authors and year & Quality of the study & Type of study & Sample & Results \\
\hline $\begin{array}{l}\text { Sousa, Pascoal, } \\
\text { Nascimento, } \\
\text { Matos, Beltrão, } \\
\text { Silva and Lopes } \\
(2016)^{(26)}\end{array}$ & $\begin{array}{l}\text { Good ( } 7 \text { out of } \\
10 \text { points in the } \\
\text { Newcastle-Ottawa } \\
\text { scale for cross- } \\
\text { sectional studies). }\end{array}$ & $\begin{array}{l}\text { Cross-sectional } \\
\text { study. }\end{array}$ & 98 patients. & $\begin{array}{l}\text { DC: } \\
\text { - Tachypnea }(30.6 \%, \mathrm{n}=30) . \\
\text { - Dyspnea }(27.6 \%, \mathrm{n}=27) . \\
\text { - Orthopnea }(25.5 \%, \mathrm{n}=25) \text {. } \\
\text { - Prolonged expiration phase }(14.3 \%, \mathrm{n}=14) . \\
\text { - Altered chest excursion }(9.2 \%, \mathrm{n}=2) . \\
\text { - Bradypnea }(7.1 \%, \mathrm{n}=7) . \\
\text { - Pursed-lip breathing }(4.1 \%, \mathrm{n}=4) . \\
\text { - Increase in anterior-posterior chest diameter }(1.0 \%, \mathrm{n}=1) \text {. } \\
\text { - Use of accessory muscles to breathe }(1.0 \%, \mathrm{n}=1) .\end{array}$ \\
\hline $\begin{array}{l}\text { Galvão, Gomes, } \\
\text { Figueiredo and } \\
\text { Bezerra }(2016)^{(27)}\end{array}$ & $\begin{array}{l}\text { Good (7 out of } \\
10 \text { points in the } \\
\text { Newcastle-Ottawa } \\
\text { scale for cross- } \\
\text { sectional studies). }\end{array}$ & $\begin{array}{c}\text { Cross-sectional } \\
\text { study }\end{array}$ & $\begin{array}{l}62 \text { patients } \\
\text { for acute } \mathrm{CHF} \\
\text { treatment. }\end{array}$ & $\begin{aligned} \text { DC: } & \\
& \text { - Dyspnea }(48.4 \%, n=30) \\
& \text { - Orthopnea }(8.1 \%, n=5) \\
\text { RF: } & \\
& \text { - Hyperventilation }(91.7 \%, n=22) \\
& \text { - Respiratory muscle fatigue }(33.3 \%, n=8) . \\
& \text { - Fatigue }(77.4 \%, n=48) \\
& \text { - Pain }(25.0 \%, n=6) \\
& \text { - Obesity }(12.5 \%, n=3)\end{aligned}$ \\
\hline
\end{tabular}

Note: $D C=$ Defining Characteristics; $R F=$ Related Factors; $C H F=$ Congestive heart failure; $P U=$ Pressure Ulcer .

Three studies describing the conceptual and operational definitions of the following defining characteristics and related factors were also identified: anxiety ${ }^{(30)}$, altered breathing depth ${ }^{(31)}$, nasal flaring $^{(10,31)}$, bradypnea ${ }^{(31)}$, decreased vital capacity ${ }^{(10,30-31)}$, increase in anterior-posterior chest diameter ${ }^{(10,31)}$, dyspnea ${ }^{(10,31)}$, altered chest excursion ${ }^{(10,31)}$, prolonged expiration phase ${ }^{(10,31)}$, decrease in inspiratory pressure ${ }^{(10,31)}$, decrease in expiratory pressure ${ }^{(10,31)}$, tachypnea ${ }^{(31)}$, use of accessory muscles to breathe ${ }^{(10,31)}$, decrease in minute ventilation ${ }^{(10,31)}$, expiratory wheeze ${ }^{(31)}$, diminished vesicular breath sounds ${ }^{(31)}$, orthopnea ${ }^{(10,31)}$, pursed-lip breathing ${ }^{(10,31)}$, adventitious breath sounds ${ }^{(30)}$ and cough ${ }^{(30)}$.

The evaluation of the methodological quality of the studies observed showed that all were considered as good according to the Newcastle-Ottawa Scale (Chart 1 and 2).

\section{DISCUSSION}

In children, the main related factor identified in the literature for the nursing diagnosis ineffective breathing pattern was bronchial secretion, which is not in the NANDA-I, followed by hyperventilation. The main defining characteristics were: dyspnea, tachypnea, cough, use of accessory muscles to breathe, orthopnea and adventitious breath sounds. Cough and adventitious breath sounds are also not included in NANDA-I definition for the diagnosis ineffective breathing pattern.

In children, the presence of the related factor bronchial secretion is related to the fact that the airway diameter is reduced in young children, which leads to narrowing of the lumen due to mucosal edema, exacerbated secretion and infiltration of inflammatory cells, leading to different degrees of obstruction. In addition, children are also unable to spontaneously expel secretions from the respiratory tract due to the immaturity of coughing mechanisms and difficulty in expectoration, causing respiratory distress ${ }^{(12,32)}$.

A study carried out with children from zero to five years old in Fortaleza (CE) identified that children with bronchial secretion have a $80.0 \%$ chance of developing an ineffective breathing pattern ${ }^{(33)}$. The presence of obstructed airways leads to excessive levels of carbon dioxide and hydrogen ions in the blood, causing hyperventilation, which is a breathing pattern deeper, faster and longer than the normal, aimed at eliminating excess carbon dioxide ${ }^{(33-34)}$.

Hyperventilation is characterized by ventilation that exceeds the metabolic demand and it is caused by two mechanisms. The first mechanism is respiratory alkalosis which, with the worsening of a primary lung disease and respiratory muscle fatigue, may lead to respiratory failure, ineffective breathing pattern and other respiratory nursing diagnoses. The other mechanism is related to direct stimulation of the respiratory center and occurs with diseases of the central nervous system, such as meningitis, hemorrhage or trauma, increasing the rate and depth of respiratory effort. Hyperventilation can also be caused by some underlying disease that causes pain, stress or anxiety. This is called psychogenic hyperventilation, and it occurs when there is no pathological organic process accounting for hyperventilation $^{(34)}$. Thus, during the physical examination, it is possible to identify hypoxemia, which is the main mechanism that leads to hyperventilation, marked by decreased oxygen saturation as measured by pulse oximetry ${ }^{(27)}$.

Regarding the defining characteristics, a study with children up to five years old showed that most of the defining characteristics were related to respiratory changes, with dyspnea present in $74.4 \%$ of the children ${ }^{(8)}$. These respiratory symptoms can be attributed to the characteristics of respiratory diseases, such as asthma and pneumonia, commonly found in children up to five years old ${ }^{(35-36)}$. Another study showed that children with dyspnea are 36 times more likely to develop an ineffective breathing pattern ${ }^{(33)}$.

A validation study identified an association between ineffective breathing pattern and acute respiratory infections, and dyspnea was found in $98.0 \%$ of the children. This defining characteristic was validated as an important finding among the signs and symptoms of acute respiratory infections. Dyspnea is cited as the most significant defining characteristic of ineffective respiratory pattern in several studies ${ }^{(12,29,37)}$. It is an important 
defining characteristic in infants with congenital heart disease, considering that approximately $99.0 \%$ of these children present this symptom in the first year of life ${ }^{(4,38)}$. It is usually caused by respiratory diseases related to secretion, infection or obstruction in the airways or by heart diseases or psychogenic factors, such as anxiety ${ }^{(34)}$.

Tachypnea, another defining feature found in our study, is generally the first manifestation of respiratory disorders in children. Tachypnea is characterized by fast, shallow breathing, more than 20 breaths per minute in adults and over 60 breaths in children ${ }^{(1)}$. It occurs due decreased compliance or respiratory muscles fatigue and is the result of pulmonary hyperventilation, which develops as an adaptive attempt of compensation ${ }^{(1,34)}$. The failure of this compensatory mechanism and the imbalance between the demand and the supply of oxygen favors the ineffective breathing pattern diagnosis ${ }^{(26)}$. The main pathologies that cause tachypnea are restrictive lung disease, hypovolemic shock, pneumonia, pulmonary edema, metabolic acidosis, septicemia, severe pain, neurological disorders and rib fractures ${ }^{(34)}$.

Regarding the defining characteristic use of accessory muscles to breathe, it should be noted that several muscles are responsible for pulmonary ventilation, and they are classified as major and accessory. The diaphragm and intercostal muscles are the main muscles involved in breathing. The accessory muscles assist when there is an extra demand for ventilation, and are represented by the scalene, sternocleidomastoid, pectoralis major and abdominal muscles. Thus, with the exacerbation or severity of respiratory dysfunction, common in chronic lung diseases, with alveolar involvement and consequent respiratory muscle fatigue, accessory muscles are required ${ }^{(34)}$. A study conducted with asthmatic children from zero to five years old in the city of Fortaleza (CE) found that children with this defining characteristic were seven times more likely to have an ineffective breathing diagnosis ${ }^{(9)}$.

Regarding cough and adventitious breath sounds, a crosssectional study conducted in Fortaleza (CE), Brazil, with 151 children aged zero to five years, showed that ineffective breathing pattern was the most prevalent diagnosis and adventitious breath sounds and cough were its most frequent defining characteristics $^{(12)}$. Cough is a response of the lower respiratory tract to the stimulation of receptors in the airways mucosa, and asthma is the most common cause in children ${ }^{(34)}$. Cough related to lung function is mainly due to airway secretions, aspiration of foreign bodies and inhalation of allergens ${ }^{(34)}$. Adventitious breath sounds, in turn, are detected in pulmonary auscultation and are the result of the passage of air through obstructed airways and the collision of air against tracheobronchial secretions ${ }^{(12)}$, which are frequent in patients with respiratory disorders. Adventitious breath sounds are wheezes (common in inspiration), rhonchi (due to accumulation of secretion), crackling, pleural friction, and crunches, which are very common in children with disorders of the respiratory system ${ }^{(34)}$.

In adults and older adults, the main related factors were fatigue, pain and obesity and the main defining characteristics were dyspnea, tachypnea and orthopnea.

Fatigue is characterized by diffuse and symmetrical weakness, involving the musculature of limbs and respiratory muscles. It is verbalized in the report of fatigue and indisposition in activities and is independently associated with prolonged mechanical ventilation, increased hospital and intensive care unit (ICU) stays and increased mortality rates ${ }^{(39)}$. Fatigue may be present in patients with different pathologies, such as cardiac, pulmonary, hematologic, oncological, as well as in patients who present pain, malnutrition and psychological symptoms such as anxiety and depression. It is more frequent among older adults and among those with greater number of comorbidities ${ }^{(40)}$. A study has shown that age, cognitive and functional status pre-ICU, concomitant diseases (mainly heart diseases) and health trajectories should also be evaluated to stratify patients with fatigue ${ }^{(41)}$. In 1998 , an American validation study with 76 patients with clinical and surgical diagnoses, with a mean age of 59 years, identified and expressed the importance of the related factors fatigue and anxiety as the cause of ineffective breathing pattern ${ }^{(17)}$.

Regarding pain, patients with this symptom may present alterations in cardiopulmonary and cerebral function, which can compromise the mechanisms responsible for cellular oxygenation and trigger a great demand to compensate them, resulting in alteration of inspiration and expiration. Other studies have also identified pain as a related factor of ineffective breathing pattern $^{(42-43)}$. Pain is an unpleasant sensation and emotional experience associated with difficult-to-manage tissue damage. The transmission of pain involves the spinal cord, which transmits the painful impulse to the brain. The transmission of pain involves the spinal cord, which transmits the painful impulse to the brain ${ }^{(44)}$. During this process, ventilation, which was previously automatically performed by the bulb, becomes voluntarily controlled by the cerebral cortex, Then, the individual begins to have artificial respirations, dyspnea and decreased airflow to the alveoli, presenting an abnormal respiratory pattern ${ }^{(45)}$.

Another related factor of ineffective breathing pattern was obesity. Several factors interfere in the respiratory mechanics of obese individuals, resulting in reduced volume and lung capacity, such as expiratory reserve volume and functional residual capacity. Excessive adipose tissue causes mechanical compression of the diaphragm, lungs and chest, which can lead to restrictive respiratory damage ${ }^{(46)}$. Obesity also reduces the complacency of the respiratory system and increases lung resistance. These factors lead to inspiratory overload, with consequent increase in respiratory work, oxygen consumption and energy cost. This can trigger the defining characteristics of the nursing diagnosis ineffective breathing pattern, such as dyspnea, fatigue, orthopnea and anxiety ${ }^{(47-48)}$.

Regarding the defining characteristic dyspnea, a study conducted in Ribeirao Preto, São Paulo, Brazil, with adult patients in the preoperative period cardiac surgery, showed that the prevalence of ineffective breathing pattern among these patients was high (70.6\%). Patients reported having a "hunger for air" or a "need to breathe more", which can be understood as dyspnea and tachypnea ${ }^{(48)}$. All patients evaluated had dyspnea and $66.6 \%$ also presented orthopnea as a defining characteristic, and $100 \%$ presented fatigue as a related factor ${ }^{(16)}$. In a study carried out in Fortaleza (CE) Brazil, dyspnea was the primary defining characteristic $^{(48)}$.

Tachypnea, orthopnea and dyspnea are very common alterations in patients with ineffective breathing pattern, due to 
respiratory muscle fatigue resulting from failure to resolve the underlying problem that led to respiratory decompensation. An imbalance between the load and the force leads to the development of progressive respiratory muscle fatigue, with consequent development of dyspnea and, later, tachypnea, in the attempt to increase the thoracic incursions to solve the problem. However, what it is observed is that respiratory muscle fatigue results in respiratory failure ${ }^{(49)}$. Respiratory fatigue resulting from not solving dyspnea and tachypnea may lead to respiratory failure and require mechanical ventilation. A study carried out in the city of São Paulo found that the defining characteristics associated with this diagnosis in patients undergoing mechanical ventilation were abnormal blood gases, respiratory rate change, hypoxemia and snoring ${ }^{(50)}$.

In view of the above, it is observed that there are clinical indicators (defining characteristics and related factors) related to the nursing diagnosis ineffective breathing pattern that are identified in the literature but not yet included in NANDA-I, 2018-2020.

\section{Study limitations}

Since it is a literature review study, the descriptors chosen may have missed articles that could have contributed to the findings of this research. However, the results found indicated new defining characteristics and related factors whose inclusion may improve the nursing diagnosis ineffective breathing pattern in the international taxonomy of nursing diagnoses.

\section{Contributions to the nursing area}

This study allowed the identification of the related factor bronchial secretion and the defining characteristics adventitious breath sounds and cough, which are not in the NANDA-I classification but can be posteriorly included, contributing to the advancement of nursing and the improvement of this nursing diagnosis in clinical practice.

These results are expected to improve care provided to patients with ineffective breathing pattern diagnosis, as it can increase awareness of different etiologies according to age group and facilitate an early diagnosis.

\section{CONCLUSION}

The present review identified the related factors and defining characteristics of ineffective breathing pattern in children, adults and older adults. Among children, the related factors were bronchial secretion and hyperventilation and the defining characteristics were: dyspnea, cough, adventitious breath sounds, tachypnea and the use of accessory muscles to breathe. It should be noted that the related factor bronchial secretion and the defining characteristics cough and adventitious breath sounds are not included in the NANDA-I classification. The high prevalence of these signs in the analyzed age group indicates the need to include them in this classification.

Among adults and older adults, the related factors identified were: fatigue, obesity and pain. The defining characteristics were: dyspnea, tachypnea and orthopnea. The related factors differed between the age groups, possibly because of the maturity of the respiratory system and the different respiratory disorders, indicating that the interventions must be directed to each related factor.

\section{FUNDING}

We thank the Acre Research Foundation (FAPAC) for granting the $\mathrm{PhD}$ scholarship from the Coordination for the Improvement of Higher Education Personnel (CAPES) and the Federal University of Acre, for granting a PhD leave of absence to conduct this research full time.

\section{REFERENCES}

1. Bettencourt ARC, Maurino IC, Prado PR, Zeitoun SS, Martins I, Leite AL. Exame do tórax: sistema respiratório. In: Barros AL (Org.) Anamnese e exame físico: diagnóstico e avaliação no adulto. 2 ed. Porto Alegre: Artmed. 2016 p. 205-35.

2. Rossi LA, Casagrande LDR. Processo de enfermagem: a ideologia da rotina e a utopia do cuidado individualizado. In: Cianciarullo TI, Gualda DMR, Melleiro MM, Anabuki MH. Sistema de assistência de enfermagem: evolução e tendências. 3a ed. São Paulo: Ícone; 2001. p. 41-62.

3. Herdman TH, Kamitsuru S. NANDA International nursing diagnoses: definitions and classification, 2018-2020. 11 th ed. New York: Thieme Publishers. 2018. $512 \mathrm{p}$.

4. Silva VM, Araújo TL, Lopes MVO. Evolution of nursing diagnoses for children with congenital heart disease. Rev Lat Am Enfermagem [Internet]. 2006 [cited 2018 Oct 28];14(4):561-8. Available from: http://dx.doi.org/10.1590/S0104-11692006000400014

5. Okuno MFP, Costa N, Lopes MCBT, Campanharo CRV, Batista REA. The most used nursing diagnoses at an emergency service. Cogitare Enferm [Internet]. 2015[cited 2018 Oct 28];20(2):383-8. Available from: doi: http://dx.doi.org/10.5380/ce.v20i2.38606

6. Whittemore R, Knafl K. The integrative review: updated methodology. J Adv Nurs. 2005;52(5):546-53. doi:10.1111/j.1365-2648.2005.03621.x

7. Wells GA, Shea B, O'Connell D, Petersen J, Welch V, Losos M, et al. The Newcastle Ottawa Scale (NOS) for assessing the quality of nonrandomized studies in meta-analyses [Internet]. Canada: Department of Epidemiology and Community Medicine, University of Ottawa; 2000 [cited 2018 Oct 28]. Available from: http://www.ohri.ca/programs/clinical_epidemiology/oxford.asp

8. Monteiro FPM, Silva VM, Lopes MVO. Diagnósticos de enfermagem identificados em crianças com infecção respiratória aguda. Rev Eletr Enferm [Internet]. 2006 [cited 2018 Oct 28];8(2):213-21. Available from: doi: https://doi.org/10.5216/ree.v8i2.7036

9. Silveira UA, Lima LHO, Lopes MVO. [Defined characteristics of the nursing diagnoses ineffective airway clearance and ineffective 
breathing pattern in asthmatic]. Rev Rene [Internet]. 2008 [cited 2018 Oct 28];9(4):125-33. Available from: http://www.redalyc.org/ html/3240/324027964015/ Portuguese.

10. Cavalcante JCBC, Mendes LC, Lopes MVO, Lima LH. [Clinical indicators of ineffective breathing pattern in children with asthma]. Rev Rene [Internet]. 2010[cited 2018 Oct 28];11(1):66-75. Available from: http://dx.doi.org/10.15253/rev\%20rene.v11i1.4476 Portuguese.

11. Chagas KLM, Lima LHO, Oliveira EAR, Luz GOA. [Nursing Diagnosis in children with signs and symptoms of asthma: a description study]. Rev Rene [Internet]. 2011 [cited 2018 Oct 28];12(2):302-8. Available from: http://www.periodicos.ufc.br/rene/article/viewFile/4206/3253 Portuguese.

12. Andrade LZC, Chaves DBR, Silva VM, Beltrão BA, Lopes MVO. Respiratory nursing diagnoses for children with acute respiratory infection. Acta Paul Enferm [Internet]. 2012[cited 2018 Oct 28];25(5):713-20. Available from: http://dx.doi.org/10.1590/S0103-21002012000500011

13. Pascoal LM, Lopes MVO, Silva VM, Beltrão BA, Chaves DBR, Santiago JMVM, et al. Ineffective breathing pattern: defining characteristics in children with acute respiratory infection. Int J Nurs Knowl. 2014;25(1):54-61. doi: 10.1111/j.2047-3095.2013.01249.x

14. Beltrão BA, Herdman TH, Pascoal LM, Chaves DBR, Silva VM. Ineffective breathing pattern in children and adolescentes with congenital heart disease: accuracy of defining characteristics. J Clin Nurs [Internet]. 2015;24(17-18):2505-13. doi: 10.1111/jocn.12838.

15. Pascoal LM, Lopes MV, Silva VM, Beltrão BA, Chaves DB, Nunes, Castro. Prognostic clinical indicators of short-term survival for ineffective breathing pattern in children with acute respiratory infection. J Clin Nurs. 2016;25(5-6):752-9. doi: 10.1111/jocn.13064

16. Pascoal LM, Lopes MV, da Silva VM, Chaves DB, Beltrão BA, Nunes MM, et al. Clinical Differentiation of Respiratory Nursing Diagnoses among Children with Acute Respiratory Infection. J Pediatr Nurs [Internet]. 2016;31(1):85-91. doi: 10.1016/j.pedn.2015.08.002

17. Carlson-Catalano J, Lunney M, Paradiso C, Bruno J, Luke BK. Martin T, et al. Clinical Validation of Ineffective Breathing Pattern, Ineffective Airway Clearance, and Impaired Gas Exchange. Image J Nurs Sch [Internet]. 1998 [cited 2018 Oct 28];30(3):243-48. Available from: https:// doi.org/10.1111/j.1547-5069.1998.tb01299.x

18. Arruda AJCG, Garcia TR. [Nursing diagnoses related to oxygenation, atributted to trauma victims admitted to ICU]. Rev Bras Enferm [Internet]. 2000 [cited 2018 Oct 28];53(3):363-74. Available from: http://dx.doi.org/10.1590/S0034-71672000000300004 Portuguese.

19. Galdeano LE, Rossi LA, Pezzuto TM. [Nursing diagnosis of patients in the preoperatory period of cardiac surgery]. Rev Esc Enferm [Internet]. 2004 [cited 2018 Oct 28];38(3):307-16. Available from: http://dx.doi.org/10.1590/S0080-62342004000300009 Portuguese.

20. Oliveira MF, Freitas MC. [Frequent nursing diagnoses and interventions for women under critical care]. Rev Bras Enferm [Internet]. 2009 [cited 2018 Oct 28]; 62(3):343-48. Available from: http://dx.doi.org/10.1590/S0034-71672009000300002 Portuguese.

21. Silva MR, Bettencourt ARC, Diccini S, Belasco A, Barbosa DA. [Nursing diagnoses for patients with acquired immunodeficiency syndrome]. Rev Bras Enferm [Internet]. 2009[cited 2018 Oct 28];62(1):92-9. Available from: http://dx.doi.org/10.1590/S0034-71672009000100014 Portuguese.

22. Lucena AF, Santos CT, Pereira AGS, Almeida MA, Dias VLM, Friedrich MA. Clinical Profile and Nursing Diagnosis of Patients at Risk of Pressure Ulcers. Rev Lat Am Enfermagem [Internet]. 2011 [cited 2018 Oct 28];19(3):523-30. Available from: http://dx.doi.org/10.1590/ S0104-11692011000300011

23. Moreira RAN, Caetano JA, Barros LM, Galvão MTG. Nursing diagnoses, related factors and risk factors during the postoperative period following bariatric surgery. Rev Esc Enferm USP [Internet]. 2013 [cited 2018 Oct 28];47(1):165-72. Available from: http://dx.doi.org/10.1590/ S0080-62342013000100021

24. Souza CC, Mata LRF, Carvalho EC, Chianca TCM. Nursing diagnoses in patients classified as priority level I and II according to the Manchester protocol. Rev Esc Enferm USP [Internet]. 2013 [cited 2018 Oct 28];47(6):1318-23. Available from: doi:10.1590/s0080-623420130000600010

25. Barros LM, Moreira RAN, Frota NM, Caetano JA. Identification of the nursing diagnoses of the cardiovascular/pulmonary responses class in patients subject to bariatric surgery. Aquichán. 2015;15(2):200-9. doi:10.5294/aqui.2015.15.2.4 Portuguese.

26. Sousa VEC, Pascoal LV, Nascimento RV, Matos TFO, Silva VM, Lopes MVO. Ineffective breathing pattern in cardiac postoperative patients: diagnostic accuracy study. Appl Nurs Res. 2016;32:134-38. doi: 10.1016/j.apnr.2016.07.005

27. Galvão PCC, Gomes ET, Figueiredo TR, Bezerra SMMS. Nursing diagnosis applied to patients with decompensated heart failure. Cogitare Enferm [Internet]. 2016 [cited 2018 Oct 28];21(2):1-8. Available from: http://revistas.ufpr.br/cogitare/article/view/44646/28162

28. Chaves DBR. Árvores de decisão para inferência de desobstrução ineficaz de vias aéreas e padrão respiratório ineficaz de crianças com infecção respiratória aguda [Dissertation on the Internet]. Fortaleza (CE): Universidade Federal de Fortaleza, Faculdade de Farmácia, Odontologia e Enfermagem, Departamento de Enfermagem; 2011 [cited 2018 Oct 28]. 96 p 2011 [cited ?]. Available from: http://www. repositorio.ufc.br/handle/riufc/3992

29. Carvalho, OMC. Medidas de acurácia dos indicadores clínicos dos diagnósticos de enfermagem respiratórios em crianças com asma [Dissertation on the Internet]. Fortaleza (CE): Universidade Federal de Fortaleza, Faculdade de Farmácia, Odontologia e Enfermagem, Departamento de Enfermagem; 2013[cited 2018 Oct 28]. 114 p. Available from: http://www.repositorio.ufc.br/handle/riufc/8500

30. Silva VM. Padrão respiratório ineficaz em crianças portadoras de cardiopatias congênitas: validação de um instrumento de avaliação dos resultados de enfermagem [Thesis on the Internet]. Fortaleza (CE): Universidade Federal do Ceará, Faculdade de Farmácia, Odontologia e Enfermagem, Departamento de Enfermagem, Programa de Pós-graduação em Enfermagem; 2007 [cited 2018 Oct 28]. 202 p. Available from: http://www.repositorio.ufc.br/handle/riufc/560

31. Avena M, Pedreira MLG, Gutiérrez MGR. Conceptual validation of the defining characteristics of respiratory nursing diagnoses in neonates. 
Acta Paul Enferm [Internet]. 2014[cited 2018 Oct 28];27(1):76-85. Available from: http://dx.doi.org/10.1590/1982-0194201400015

32. Sociedade Brasileira de Pneumologia e Tisiologia. IV Diretrizes brasileiras no manejo da asma. J Bras Pneumol [Internet]. 2012 [cited 2018 Oct 28];32(Supl 7):S447-S474. Available from: http://dx.doi.org/10.1590/S1806-37132006001100002

33. Silva VM, Lopes MVO, Araújo TL. [Odds ratio for nursing diagnoses in children with congenital heart disease]. Invest Educ Enferm [Internet]. 2007[cited 2018 Oct 28];25(1):30-8. Available from: http://www.redalyc.org/articulo.oa?id=105215404003 Portuguese.

34. Kliegman RM, Stanton BF, Geme J St, Schor NF, Berhman RE. Nelson Tratado de Pediatria. 19 ed. Rio de Janeiro: Elsevier; 2014. Chapter 52: Deficiência de vitamina $\mathrm{E}$.

35. Silva VM. Caracterização de diagnósticos de enfermagem em crianças com cardiopatias congênitas: estudo num hospital especializado em doenças cardiopulmonares [Dissertation on the Internet]. Fortaleza (CE): Universidade Federal do Ceará, Faculdade de Farmácia, Odontologia e Enfermagem, Departamento de Enfermagem, Programa de Pós-Graduação em Enfermagem;. 2004 [cited 2018 Oct 28]. 119 p. Available from: http://www.repositorio.ufc.br/handle/riufc/556

36. Ewert R, Gläser S. Dyspnea. From the concept up to diagnostics. Internist. 2015;56(8):865-71. doi:10.1007/s00108-015-3690-3

37. Hockenberry MJ, Wilson D. Wongs Essentials of pediatric nursing. 8th ed. Louis: Mosby; 2008. 1064 p.

38. Behrman RE, Kliegman RM, Jenson HB. Tratado de pediatria. Rio de Janeiro (RJ): Guanabara Koogan; 2002. Parte XX: Sistema cardiovascular. p. 1318-433.

39. Latronico $\mathrm{N}$ e Bolton CF. Critical illness polyneuropathy and myopathy: a major cause of muscle weakness and paralysis. Lancet Neurol [Internet]. 2011 [cited 2018 Oct 28];10(10):931-41. Available from: https://doi.org/10.1016/S1474-4422(11)70178-8

40. Smeltzer SC, Bare BG, Hinkle JL, Cheever KH. Brunner \& Suddarth: Tratado de Enfermagem Médico Cirúrgica. 12 th ed. Rio de Janeiro: Guanabara Koogan; 2012. 2 vols.

41. Latronico N, Herridge M, Hopkins RO, Angus D, Hart N, Hermans G, et al. The ICM research agenda on intensive care unit-acquired weakness. Int Care Med [Internet]. 2017[cited 2018 Oct 28];43(9):1270-81. Available from: https://doi.org/10.1007/s00134-017-4757-5

42. Grupo Brasileiro de Classificação de Risco. Diretrizes para implementação do sistema Manchester de classificação de risco nos pontos de atenção às urgências e emergências [Internet]. 2 ed. Belo Horizonte (MG): Grupo Brasileiro de Classificação de Risco; 2015 [cited 2018 Oct 28]. 18 p. Available from: http://gbcr.org.br/public/uploads/filemanager/source/54c127352e3b2.pdf

43. Ferreira F, Andrade J, Mesquita A, Campello G, Dias C, Granja C. The emergency room-analysis and evaluation of an organizational model. Rev Port Cardiol [Internet]. 2008 [cited 2018 Oct 28];27(7-8):889-900. Available from: https://www.ncbi.nlm.nih.gov/pubmed/18959087

44. Freitas EV, Py L. Tratado de geriatria e gerontologia. Rio de Janeiro: Guanabara Koogan; 2016. 1679 p.

45. Atkinson LD, Murray ME, Fonseca AV. Fundamentos de enfermagem. Rio de Janeiro: Guanabara Koogan; 1989. 638 p.

46. Paisani DM, Chiavegato LD, Faresin SM. Lung volumes, lung capacities and respiratory muscle strength following gastroplasty. J Bras Pneumol [Internet]. 2005[cited 2018 Oct 28];31(2):125-32. Available from: http://dx.doi.org/10.1590/S1806-37132005000200007

47. Dal Sasso GTM, Barra DCC, Paese F, Almeida SRW, Rios GC, Marinho MM, et al. Computerized nursing process: methodology to establish associations between clinical assessment, diagnosis, interventions, and outcomes. Rev Esc Enferm USP [Internet]. 2013[cited 2018 Oct 28];47(1):238-45. Available from: http://dx.doi.org/10.1590/S0080-62342013000100031

48. Rocha LA, Maia TF, Silva LF. [Nursing diagnoses in patients outgoing cardiac surgery]. Rev Bras Enferm [Internet]. 2006 [cited 2018 Oct 28];59(3):321-26. Available from: http://dx.doi.org/10.1590/S0034-71672006000300013 Portuguese

49. Ferreira EVM. Respiratory muscles: myths and secrets. J Bras Pneumol [Internet]. 2015[cited 2018 Oct 28]; 41(2):107-9. Available from: http:// dx.doi.org/10.1590/S1806-37132015000200002

50. Zeitoun SS, de Barros AL, Michel JL, de Bettencourt AR. Clinical validation of the signs and symptoms and the nature of the respiratory nursing diagnoses in patients under invasive mechanical ventilation. J Clin Nurs. 2007;16(8):1417-26. doi:10.1111/j.1365-2702.2006.01632.x 